\title{
Blended Electronic Learning Readiness in Riyadh City High School
}

\author{
Walid Qassim Qwaider \\ College of Science and Humanities in Ghat \\ Majmaah University, Saudi Arabia
}

\begin{abstract}
This paper examines the suitability of applying blended electronic learning at High School MANAR SDEER Special (HSMSS) in Riyadh City through analyzing students' views on blended learning environment. Saudi High School began to move with the international trend towards blending and faceto-face with online help when developing new educational processes.This study is to describe the opinions of the realizations of students in learning styles and blended electronic learning Riyadh city high school. The study was carried out on (50) students in High School MANAR SDEER Special (HSMSS) in Riyadh City and pedagogical design to assessment courses 1433-1434H fall semesters. Researcher through a questionnaire to determine the status of students High School MANAR SDEER Special (HSMSS) opinions on blended electronic learning and its performance process; open questions develop to be managed students in sometimes; some students achieve, and records, prove to students participating Internet environment, and the data collected for this study.
\end{abstract}

The results indicated that the more the level of educational attainment for students a positive way towards traditional education face-to-face more interested by the students, the highest score mean $=8.01$, and offset directly from this process Electronic learning (EL) (7.88) the combination of these two styles of education resulting in a blended electronic learning (EL) after reaching import content (7.83) Assessment Report 7.86. With the increased interest in the context of adopting blended electronic learning approach at secondary education environment, this work may be of interest to those engaged in conducting research training as well as other stakeholders in the academe to improve the conduct of action research in their respective educational.

\section{Keywords}

Blended Electronic learning, Electronic learning, Learning styles, face-to-face interaction, adoption blended electronic learning SMSS, Riyadh City.

\section{INTRODUCTION}

The concept is used Blended Electronic Learning often focused on the ministry of higher education and the ministry. American Society of Training and Development explained blended Electronic learning are one of the top ten directions emerging in the knowledge manufacture extradition [11]. In 2002, the ministry of higher education quoted of the University of Pennsylvania as the confluence of between online education and classing was seeing "the single biggest forward in High School is recognized today." In that article and quoted editor asynchronous learning networks forecasting a significant increase in the number of blended learning courses in the ministry of higher education include $80 \%$ to $90 \%$ of each semester [5].
The concept Blended Electronic Learning are varied. For example, blended electronic learning can be incorporated into a mixture of Web-based and traditional learning. It gathers between media and tools deployed in the electronic learning environment to achieved advantages both Web-based and traditional learning [4].

Application of blended electronic learning in secondary schools in Saudi Arabia its attention from the ministry of education. Application blended electronic learning is one of the key challenges facing high school in Saudi Arabia and move the problem to universities. The Ministry of Education understands on the need to integrate Web-based education with traditional education to overcoming barriers in this problem [11]. The main problem definition here is how can to develop blended electronic learning in secondary schools in Riyadh city.

\section{LITRATURE REVIEW}

A lot of testing blended electronic learning style for High School education. the research also suggests that blended electronic learning is not really new phases of education and learning in the ministry of education, considers the tools used on the Internet that can facilitate communication and interaction and collaborative learning between the learner and the teacher that the process is easy to use and navigate through them the tools.

Fein and Bucceri (2008) explain blended electronic learning environment that integrates the positives of electronic learning approach with some aspects of useful traditional learning, such as face-to-face interaction. Blended electronic learning pool traditional physical layers (face to face) with some tools of virtual learning integrates [7].

Dziuban et al. (2004) Took the survey in a U.S. university found that blended electronic courses could ability to develop learning results while reduction precipitation rates compared with electronic learning courses (online), so claimed that the blended electronic learning similar to, or, teaching and faceto-face pure [6].

In Lee and Chan (2007) surveys of group work of students and the outcome indicated that students best to learn blended phase, which keep some student of face-to-face interaction teaching and the use of electronic learning together. Each of these actions has attention on higher education with the university environment preparation [10].

Franklin van Harmelen (2007) presented four prestigious universities in the Britain justification and means for the application of Web 2.0 tools in the education process. The study pointed out Web 2.0 will have an effect on the students and instructor in the field of formal education, a basis for action and for a lifetime [8]. 
Richard et al, (2009) in this study, provide instruction for the content design of learning, trans boundary cooperation learning system called blended learning (BL) open origin mathematician and science related with partnership network held for its implementation in deferent countries. blended learning is to develop a large, virtual classroom free video audio units BL secondary education math subject and science virtual classes between teachers and learners, virtual classes established by professional (volunteer) teachers from all of the world and is aims to submitting industrialization likely practice that will developed teaching skills and maintain learners "benefit in mathematician and science[12].

Steve (2008) illustrates the objectives and benefits of different learning strategies with a focus on blended electronic learning and attention-Mail cognitive skills through a cooperative electronic learning environment, the design of the contents of a systematic study of the geometric models "blended electronic learning portal [13].

Akkoyunlu Soylu (2006) explain the fast development in the use of educational technology, especially focusing on the use of education-Mail applications on the Internet, and telecommunications that showed many of the growing interest in the methods of learning-Mail to talk for their students. The purpose of this study is to examine the kinds of learning in students and their views on the blended electronic learning [3].

Our study consideration on the context in the adoption of the blended electronic learning approaches in secondary education environment for secondary male schools Manar Sadir. In spite of the limitations of this study was to determine the Mannar A sudair secondary school of the region Riyadh in the "Majmaah city", so as to reveal the results expressed the view that blended electronic learning environment technically capable of mix learning adoption and they are ready to accept this form of modern blended electronic learning.

\section{RESEARCH PROBLEM AND MOTIVATION}

Blended electronic learning supports all the advantages of electronic learning including a decline in costs, time competence, and comfort for the student so a basic realization of face-to-face interaction. To the other side, blended electronic learning focused on enhancing the educational aims through the implementation of student "right" learning techniques to watch the "right" student learning kind to transfer the "right" student skills " suitable " time "right".

The merge electronic learning with traditional learning environment produces blended electronic learning that combines the beneficial aspects of the positive environment for both methods. E-learning system to ensure flexibility and efficiency that cannot be found in a classroom environment, while face-to-face social interaction offers an educational environment that is needed to learn. Definitions vary from one institution to another, known as blended electronic learning, in this paper mainly as a combination of face-to-face and on the Internet called blended electronic learning, Key research questions that motivated our study are:

1. What are opinions High School MANAR SDEER Special (HSMSS) in Riyadh city on blended electronic learning?

2. Are there opinions High School MANAR SDEER Special (HSMSS) in Riyadh city on blended electronic learning in respect of their implementation level?

3. What are opinions High School MANAR SDEER Special (HSMSS) in Riyadh city on blended electronic learning of their frequency of sharing to the forum?

\section{RESEARCH OBHECTIVS}

The specific objectives of this study are:

1. To characterize the students opinions on the adopting blending electronic learning approach.

2. To develop a perception of the barriers and challenges that students faced, and the ways their quest to confront these problems.

3. To confirmation, principles through which adopting blending electronic learning can be enabled and guided by secondary education climate, it took the learners High School MANAR SDEER Special (HSMSS).

4. To determine if adopting blending electronic learning readiness in High School MANAR SDEER Special (HSMSS), and can be the advantage to go universities.

\section{BLENDED ELECTRONIC LEARNING}

Has become one of the main age requirements in order to change the priorities and requirements of the education of the learner to another, and so must the organizations and institutions that use the methods of learning a combination in the strategies of learning to get the right content and the form and time appropriate for individuals, and it includes mix learning and multimedia rendering, and are designed to complement each other, and enhance the learning behavior and its application. Mixed education programs may include multiple forms of learning tools, such as cooperative programs or default direct, electronic and decisions adopted speed on the same learner, systems and electronic performance ancillary support in the built environment to work tasks, systems and learning management, and synthesizes mix learning different activities relies on educational events, including traditional classroom (face-to-face) and e-education simultaneous, speed and self-learning (adopted at the same speed on the learner). At the most basic level of education experience gathered mixed between direct forms of online learning and indirect, and usually means direct mail online education "use of the Internet and intranet," while the indirect education is occurring within the traditional rows [2].

Advantages Blended learning: Can identify the most important features blended electronic learning as explain $\mathrm{Al}$ Khan, including the following [2]:

Improve the effectiveness of education and by providing more harmony and harmony between the learner requirements and tutorial provided.

Expand the extent of access: The adoption of a method to provide only one, inevitably determines the pictures in addition, patterns of access to the educational program, or the transfer of knowledge, while the mixture education model allows multiple images to reach learners.

Increase the effectiveness of utilization of costly education 
programs: The integration of different submission methods lead to take advantage of the programs offered, The programmail needs to be expensive, but provided through virtual educational sessions and integrated Resume material speed and simple, such as documents, case studies, and the facts recorded for education, and appointments scripts and presentations, you may equivalent to the same cost.

\section{ELECTRONIC LEARNING IN RIYADH CITY HIGH SCHOOL}

Among the important objectives of the policies of education in the Kingdom of Saudi Arabia is taking with the latest and the consolidation of these lofty goals and in line with the evolution, the technology in the world accelerated in the field of information technology that has become the most important tools of development at the present time has been computerization curriculum at public schools in the 1984/1985 school year within the secondary education developer program, which at the time was applied to three articles and form, is the computer introduction, the programming language BASIC, information systems. In 1990 stopped work in secondary education developer curricula of the three teaching phase secondary to the various departments. In 1993 his new section has been added to the secondary stage called the Science and Technology Department has been its own curriculum design, but it is limited under the terms of non-proliferation has not been applied only in a limited number of schools because of the cost of operation and requirements. In his 1995/1996 has been increasing the number of shares of the computer course to become, and calculating the practical side of the material from within the exam, two servings a week at the secondary level where the basic examinations were computer material before it is examined only in theory over the previous ten years. Based on the recommendations of the National Family Computer 1998/1999 has been modified computer platforms, which have been applied in a modified H-grade SECOND 1999/2000 first secondary and modified by e been applied to the second grade. Teaching computer subject of the theory of the evolution of the stage to the stage of the process and applied, which means that it will create a generation capable of innovation in the field of computers, and this development to know what is the computer? Moreover, how does it work? Make thinking e-education requirement in supplying the educational process more modern technology until it became the term e-learning word-students, teachers, school administrations and parent's modern education [1].

\section{RESEARCH METHODOLOGY}

The objective of this study is to describe the opinions of the realizations of students in learning styles and blended electronic learning Riyadh city high school.

Researcher through a questionnaire to determine the status of students High School MANAR SDEER Special (HSMSS) opinions on blended electronic learning and its performance process; open questions develop to be managed students in sometimes; some students achieve, and records, prove to students participating Internet environment, and the data collected for this study.

The study was carried out on (50) students in High School MANAR SDEER Special (HSMSS) in Riyadh City and pedagogical design to assessment courses 1433-1434H fall semesters.

The repetitions of sharing to the actions recorded and their missions that sent to the actions saved, and the repetitions of sharing recorded by student names. Sharing frequency in a process was measured over a 16-week period. The participation frequency scale is as follows: "12-18: High", "611: Medium", 0-5: Low."

The developed a questionnaire to determine the views students High School MANAR SDEER Special (HSMSS) ready 'blended electronic learning performance and consists of (50) material. The students were asked to rate each item on a scale ranging from 1-10. Considered grades obtained as follows: "1-5: Low,"5.01-7: Average", "7.01-10: high." Reliability coefficient was calculated the alpha size of 0.72 . I took opinions and overtures from expert subject mind about content validation instrument.

The assessment student's collection of student scores, course duties, sub scores acquire through midterm through exams, studies during the process, at the last semester and their effort in the operation were taken into account to assessment their attainable level. The performance levels were conceded as follows: "84.01 - 100: High", "65.01 - 84: Medium", "0 - 65: Low".

\section{RESULTS AND DISCUSSION}

In this section discussion statistical analysis by answering the research questions:

\section{i. What are opinions HSMSS in Riyadh city on blended electronic learning?}

The table (1) explain means general notes it was received from the questionnaire agree with 7.21 and the highest score mean $(\overline{\boldsymbol{x}}=8.01)$ containable with to a face-to-face interaction side of the process. Degrees are ranking as mentioned above as follows: "1-5: Low,"5.01-7: Average", "7.01-10: high." In addition to, this outcome can be an illustration in several ways: face-to-face interaction between (learners and teachers). In addition, have face-to-face interaction to be more identification courses to study the predilection of learners, and able to meet the aspirations of students in face-to-face interaction in study meeting, for example, can be had learners are able to find all the subjects which have difficulty in accordance.

Table 1. Opinions High School MANAR SDEER Special (HSMSS) in Riyadh city on blended electronic learning

\begin{tabular}{|c|c|c|c|}
\hline Items & Number & $\bar{x}$ & SS \\
\hline Ease of connecting with other platforms & 50 & 7.57 & 1.64 \\
\hline Electronic learning (EL) & 50 & 7.88 & 1.67 \\
\hline Import Content & 50 & 7.83 & 1.72 \\
\hline Face-to-face Interaction & 50 & 8.01 & 1.65 \\
\hline Assessment Report & 50 & 7.86 & 1.63 \\
\hline Blended Electronic Learning & 50 & 7.60 & 1.09 \\
\hline Inclusive & 50 & 7.21 & 1.22 \\
\hline
\end{tabular}


The Table (1) shows one of the hallmarks of most education categories remote is the absence of face-to-face social interaction between learners and teachers. The results showed that face-to-face interaction is necessary for students. Besides, the reports showed the students and their comments reflect the view that the blended of learning opportunities for enhanced learning. A poll demanded to express my deep appreciation for the provision of built-positive education and evaluation of learning.

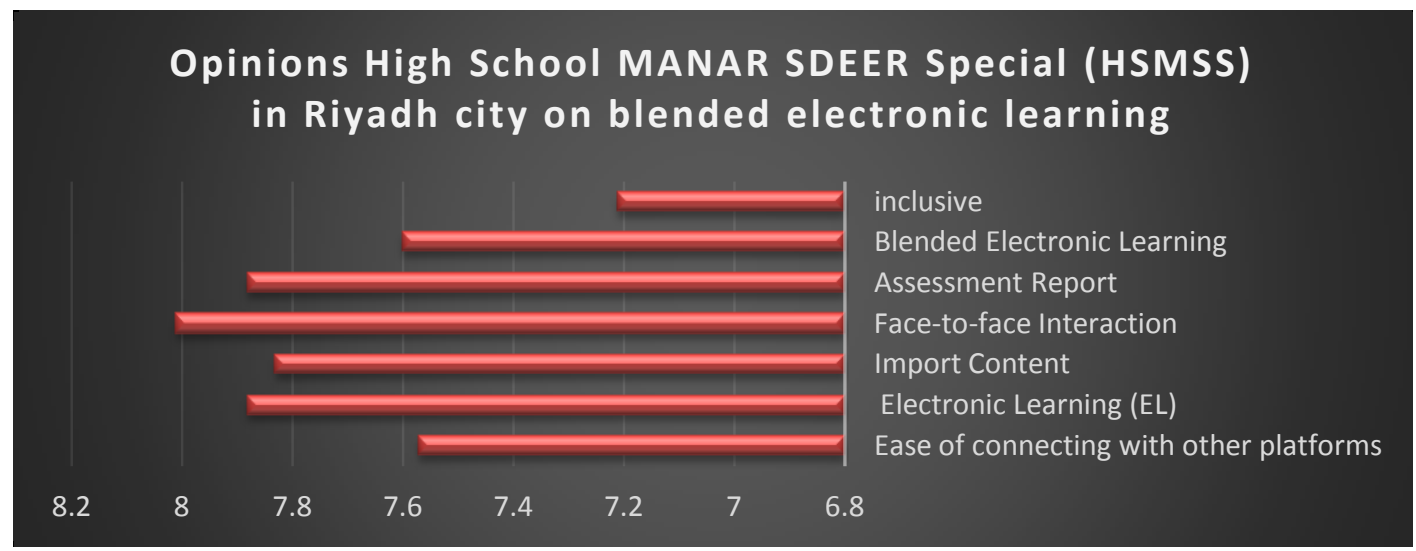

Figure 1. Opinions High School MANAR SDEER Special (HSMSS) in Riyadh City on Blended Electronic Learning

The Figure (1) shows one of the hallmarks of most education categories remote is the absence of face-to-face social interaction between learners and teachers. The results showed that face-to-face interaction is necessary for students. Besides, the reports showed the students and their comments reflect the view that the blended of learning opportunities for enhanced learning. A poll demanded to express my deep appreciation for the provision of built-positive education and evaluation of learning [1]. ii. Are there opinions HSMSS in Riyadh city on blended electronic learning in respect of their implementation level?

The table (2) shows the opinions HSMSS in Riyadh city on both blended electronic learning process application and blended electronic learning in the change with a higher level of performance.

Valuation method in this table (2) differs from previous methods, as there are some difficulties, and produces a biweekly meeting of these difficulties. It is not well to be learner's front of learning by the on-line learning.

Table 2. Opinions HSMSS in Riyadh city on blended electronic learning in respect of their implementation level

\begin{tabular}{|c|c|c|c|c|c|c|}
\hline & \multicolumn{2}{|c|}{ Low } & \multicolumn{2}{|c|}{ Medium } & \multicolumn{2}{|c|}{ High } \\
\hline & $\bar{x}$ & ss & $\bar{\pi}$ & ss & $\bar{\pi}$ & ss \\
\hline Items & \multicolumn{2}{|c|}{10} & \multicolumn{2}{|c|}{15} & \multicolumn{2}{|c|}{25} \\
\hline Ease of connecting with other platforms & 4.98 & 1.77 & 7.7 & 1.01 & 8.63 & 0.66 \\
\hline Electronic learning (EL) & 5.38 & 1.63 & 7.15 & 1.15 & 9.01 & 0.59 \\
\hline Import Content & 4.66 & 0,86 & 7.47 & 1.21 & 8.96 & 0.55 \\
\hline Face-to-face Interaction & 5.09 & 1.57 & 7.75 & 1.05 & 9.03 & 0.54 \\
\hline Assessment Report & 4.97 & 0.44 & 7.58 & 1.18 & 8.93 & 0.77 \\
\hline Blended Electronic Learning & 6.18 & 0.79 & 6.94 & 0.79 & 8.40 & 0.49 \\
\hline Inclusive & 4.87 & 0.52 & 6.82 & 0.47 & 8.14 & 0.26 \\
\hline
\end{tabular}

In fact, when the teacher education process and explain the decision in comparison with other teaching aids, it is logic that the student chooses education and face-to-face interaction with the teacher and both competitive and taking more interest from other Allow sail. Thus, it can be a chance to succeed best if I had more interaction, education, face to face, and this coincides with the opinion of Garrison and Kanuka, 2004, compared to the blended learning environment of traditional learning environment, and pointed out that learning more effectively and efficiently happens in the non-blended environment to raise the success rate of the number of students [9]. Environment to raise the level of student success. 


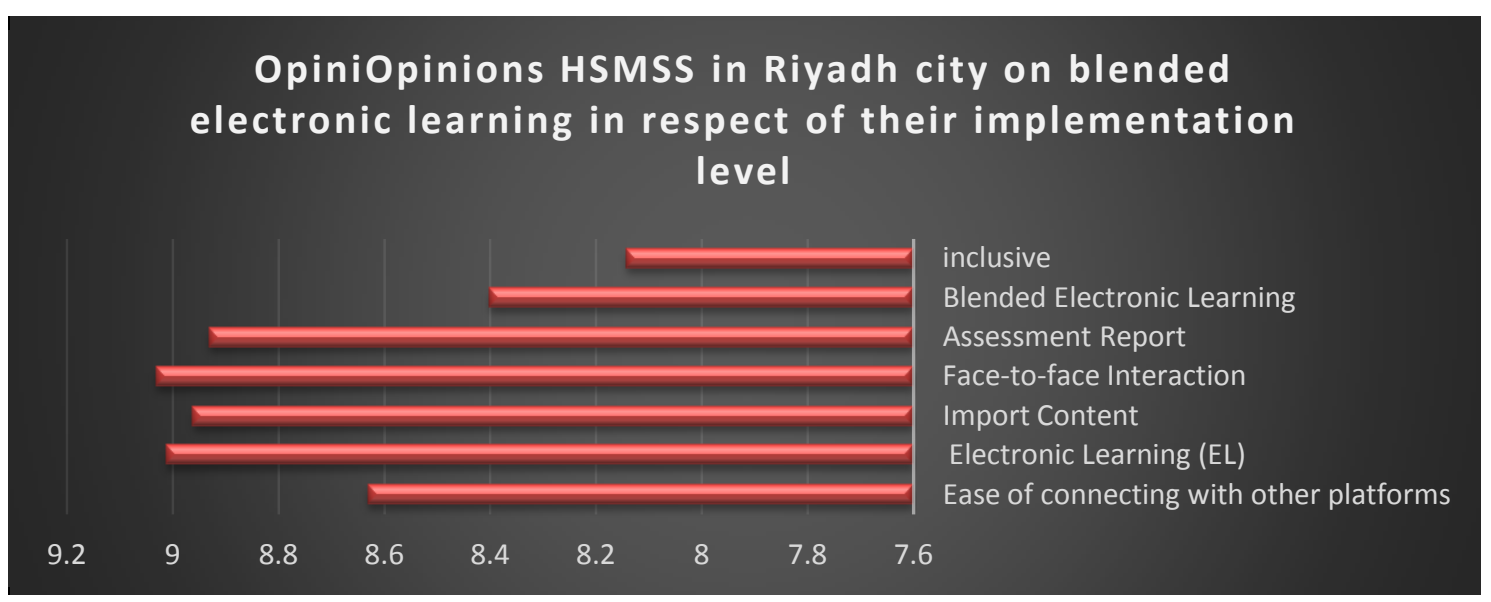

Figure 2. Opinions HSMSS on blended electronic learning in respect of their implementation level

The Figure (2) shows the most education categories classes gained significant comparative importance and took the highest rate (9.03), are: Face-to-face Interaction, while we find that the only one who got the relative importance of the less variable (8.14) is: Inclusive.

iii. What are HSMSS in Riyadh city on blended electronic learning of their frequency of sharing to the forum?

The table (3) shows students have clear interests in face-to- face interaction (9.05) in this study. He pointed out that the points of view of the students in frequency electronic learning (EL) (9.03) implementation process, learning in a mixed change in the density of the environment increase participation.

Then followed by the import content, assessment report, Ease of connecting with other platforms, Blended Electronic Learning and finally Inclusive.

Table 3. Opinions HSMSS in Riyadh city on blended electronic learning of their frequency of sharing to the forum?

\begin{tabular}{|c|c|c|c|c|c|c|}
\hline & \multicolumn{2}{|c|}{ Low } & \multicolumn{2}{|c|}{ Medium } & \multicolumn{2}{|c|}{7} \\
\hline & $\bar{x}$ & ss & $\bar{x}$ & ss & $\bar{x}$ & ss \\
\hline Items & \multicolumn{2}{|c|}{10} & \multicolumn{2}{|c|}{10} & \multicolumn{2}{|c|}{30} \\
\hline Ease of connecting with other platforms & 5.30 & 1.00 & 7.45 & 0.76 & 8.63 & 0.66 \\
\hline Electronic learning (EL) & 5.76 & 1.61 & 7.36 & 1.01 & 9.03 & 0.59 \\
\hline Import Content & 5.14 & 1.13 & 7.94 & 0.89 & 8.97 & 0.54 \\
\hline Face-to-face Interaction & 5.77 & 1.77 & 7.95 & 0.84 & 9.05 & 0.54 \\
\hline Assessment Report & 5.50 & 1.00 & 7.88 & 1.06 & 8.92 & 0.78 \\
\hline Blended Electronic Learning & 6.25 & 0.65 & 7.14 & 0.83 & 8.39 & 0.49 \\
\hline Inclusive & 5.25 & 0.72 & 7.06 & 0.26 & 8.14 & 0.25 \\
\hline
\end{tabular}

The Import content (9.05) and understandable terms of use and display Ease of connecting with other platforms (8.14). Environment Forum at the Ease of connecting with other platforms is very benefits for the retrieval of information and relate to database management. Sharing my thoughts on the track with my students and teachers in a limited Environment forum collaboration to learning .
They develop our learning through lectures and profits the right style and we can study every day. The web page can exchange anything in my mind with my teacher or my colleagues. In my opinion, the way the online environment and face-to-face are a suitable environment is very effective [3]. 


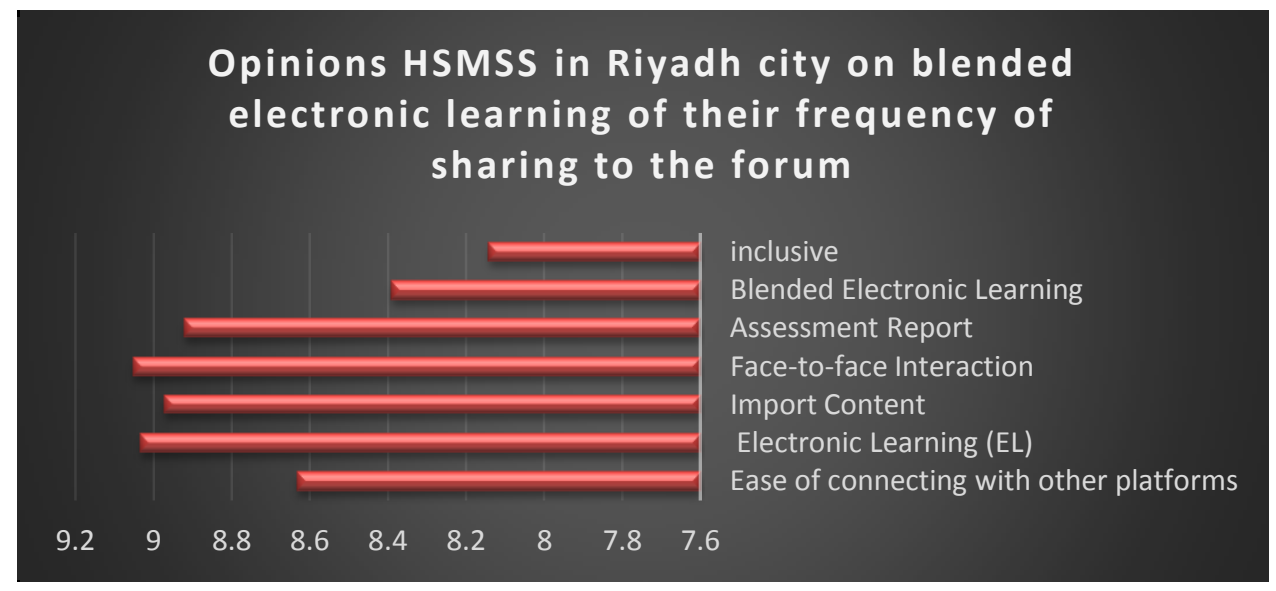

Figure 3. Opinions HSMSS on Blended Electronic Learning of their Frequency of Sharing to the Forum

The Figure (3) shows the most education categories classes gained significant comparative importance and took the highest rate (9.05), are: Face-to-face Interaction, while we find that the only one who got the relative importance of the less variable (8.14) is: Inclusive.

\section{CONCLUSION}

The results indicated that the more the level of educational attainment for students of MANAR SDEER Special (HSMSS) their participation in the forum and the school environment in a positive way towards traditional education face-to-face more interested by the students, the highest score mean $=8.01$, and offset directly from this process Electronic learning (EL) (7.88) the combination of these two styles of education resulting in a blended electronic learning (EL) after reaching import content (7.83) Assessment Report 7.86. Implement the Blended Electronic Learning, as mentioned above positive views about the increasing blended electronic learning commensurate with the increased frequency of participation in the forum to emphasize the importance of communication and interaction in the effectiveness of electronic learning. The study teaching and face-to-face interaction highlighted, and contributed to the use of this method in the forum for student learning. Students who have a low level of achievement, and stated that he is not familiar with the use of the I Electronic learning (EL) stresses the importance of making students aware of these environments through learning style blended electronic learning before the adoption of the method, which is based entirely on electronic learning and support web page courses with face-to-face interaction.

\section{REFERENCES}

[1] Abdul Karim, Mashaal. (2008). The reality of the use of e-learning in the UK National Schools in Riyadh, Master Thesis in the Faculty of Education, Department of means and Technology Education, King Saud University, pp.24.

[2] Al Khan. Badr . (2005). E-learning strategies. Translation Ali al-Moussawi and others. Syria . Dar Shuaa.

[3] Akkoyunlu, B., \& Yılmaz, M. (2006). Türetimci çoklu ortam ögrenme kuramı [Generative multimedia learning environment]". Hacettepe University, Journal of Education, 183-193.
[4] Arbaugh J.B. (2005). How much does "subject matter" matter?, A study of disciplinary effects in on-line MBA courses, Academy of Management Learning \& Education, 4(1), pp. 57-73.

[5] Bonk C. J. and Graham C. R. (2004). Blended Learning Systems: Definition, Current Trends, and Future Direction, by John Wiley \& Sons, Inc. Published by Pfeiffer - An Imprint of Wiley, 989 Market Street, San Francisco, CA 94103-1741.

[6] Dziuban, C.D., Hartman, J.L., Moskal, P.D. (2004). Blended learning. EDUCAUSE Center for Applied Research Bulletin, 7, 112. Volume 2004, Issue 7.

[7] Fein, A., \& Bucceri, M. (2008). "A case study approaches to blended learning". Retrieved January 15.

[8] Franklin, T. \& van Harmelen, M. (2007). Web 2.0 for Content for Learning and Teaching in Higher Education.

[9] GARRISON, D. R., \& Kanuka, H. (2004). Blended learning: Uncovering its transformative potential in higher education. Internet and Higher Education, 7, 95105 .

[10] Lee, P. W. R. and Chan, F. T. (2007). Blended Learning: Experiences of Adult Learners in Hong Kong. In: Fong, J., Wang F.L. (Eds) Blended Learning, pp. 79-87, Pearson.

[11] Qwaider, Walid. (2010). Blended Learning System Using Building Artificial Intelligent Tutoring Model: An Empirical Study, University of Banking and Financial Sciences, Jordan, Amman.

[12] Richard C. Larson, M. Elizabeth M. (2009). Open Education resources for Blended Learning in High Schools: Overcoming Impediments in Developing countries, Journal of Asynchronous Learning Networks, Volume 12: Issue 1.

[13] Steve Y. W. Lam. (2008). Blended e-Learning Model for Geometrics Curriculum: Design, Implementation, and Evaluation. Hong Kong SAR, China, Enschede. 